\title{
Simulation of observed climate changes in 1850-2014 with climate model INM-CM5
}

\author{
Evgeny Volodin and Andrey Gritsun \\ Institute for Numerical Mathematics, INM RAS, Gubkina 8, Moscow 119333, Russia \\ Correspondence: Evgeny Volodin (volodinev@gmail.com) \\ Received: 10 April 2018 - Discussion started: 8 May 2018 \\ Revised: 4 October 2018 - Accepted: 8 October 2018 - Published: 25 October 2018
}

\begin{abstract}
Climate changes observed in 1850-2014 are modeled and studied on the basis of seven historical runs with the climate model INM-CM5 under the scenario proposed for the Coupled Model Intercomparison Project Phase 6 (CMIP6). In all runs global mean surface temperature rises by $0.8 \mathrm{~K}$ at the end of the experiment (2014) in agreement with the observations. Periods of fast warming in 1920-1940 and 1980-2000 as well as its slowdown in 1950-1975 and 2000-2014 are correctly reproduced by the ensemble mean. The notable change here with respect to the CMIP5 results is the correct reproduction of the slowdown in global warming in 2000-2014 that we attribute to a change in ocean heat uptake and a more accurate description of the total solar irradiance in the CMIP6 protocol. The model is able to reproduce the correct behavior of global mean temperature in 19802014 despite incorrect phases of the Atlantic Multidecadal Oscillation and Pacific Decadal Oscillation indices in the majority of experiments. The Arctic sea ice loss in recent decades is reasonably close to the observations in just one model run; the model underestimates Arctic sea ice loss by a factor of 2.5. The spatial pattern of the model mean surface temperature trend during the last 30 years looks close to the one for the ERA-Interim reanalysis. The model correctly estimates the magnitude of stratospheric cooling.
\end{abstract}

\section{Introduction}

Noticeable climate changes were observed during the last century. The main feature of these changes is global warming and it is widely accepted that its most probable cause is an increase in the anthropogenic greenhouse gas concentration (Bindoff et al., 2013). The nature of several other important changes is not as clear and is still under discussion. Global warming was not uniform in time. There are two wellknown periods of acceleration in 1920-1940 and 1980-2000 and two periods with a stabilization of the global mean temperature in 1950-1975 and 2000-2014.

The reason for this oscillatory behavior is still debated. In Wilcox et al. (2013) it is shown that the period of climate stabilization in 1950-1975 can be connected with the increase in anthropogenic $\mathrm{SO}_{2}$ emissions in Europe and North America, as well as with stratospheric volcanic eruptions (Bindoff et al., 2013), while the decrease in warming in 2000-2014 could be attributed to a slowdown of methane and the tropospheric ozone concentration increase rate. On the other hand, the ensemble of CMIP5 model runs (with all the mentioned aspects of aerosol and greenhouse gas forcing taken into account) continues to raise global temperature in 2000-2014 albeit at a slower rate (Checa-Garcia et al., 2016).

Another point of view on this problem is that the acceleration and deceleration of global warming could be a manifestation of internal climate variability with a timescale of 60-70 years (Meehl et al., 2011). The Atlantic Multidecadal Oscillation (AMO) and Pacific Decadal Oscillation (PDO) are the most known drivers of internal variability in the climate system on multidecadal timescales. Indeed, Dong and McPhaden (2017) showed the importance of AMO-like and PDO-like internal variability for local temperature in the North Atlantic and the North Pacific but questioned its ability to produce a significant anomaly in global mean temperature. A connected question is to what extent the observed long-term variability of the AMO and PDO patterns is an internal process or is forced by some external factors. There is some evidence (Ting et al., 2014) that negative values of the 
AMO index in 1950-1970 could be attributed to enhanced $\mathrm{SO}_{2}$ emissions in Europe and North America.

One of the most intriguing features of recent climate changes is a rapid decrease in Arctic sea ice area since year 2000 coupled with strong Arctic warming. Similar Arctic warming was also observed in the middle of the 20th century. The ensemble of CMIP5 models underestimates the amount of sea ice loss in the 2000s by a factor of 2 (Bindoff et al., 2013; Stroeve et al., 2012). The INMCM4 (Volodin et al., 2013) that participated in CMIP5 also strongly underestimates Arctic sea ice loss extent in the beginning of the 21st century. On the other hand, the INMCM4 (and other CMIP5 models; Stroeve et al., 2012) demonstrates a loss of Arctic sea ice of comparable magnitude at different times (in the middle of the 20th century for INMCM4). Moreover, a similar sea ice loss event was produced by INMCM4 during a preindustrial control run. So, the question is to what extent the 21st century Arctic sea ice degradation is due to internal climate variability and whether the next generation of models with the new CMIP6 forcing recommendations will be able to reproduce sea ice changes in the beginning of the 21 st century.

Regional climate changes during the last several decades also show some interesting features. For example, in 20002014 there is almost no winter warming in the majority of Eurasia with respect to the previous decades and a small cooling was even observed in some places. One possible reason could be the response of atmospheric dynamics to Arctic sea ice loss (Overland et al., 2011). However, this hypothesis is questioned by other studies (see McCuscer et al., 2016, as an example).

The aim of this study is to analyze basic features of climate changes during 1850-2014. The data for the analysis (ensemble of seven historical runs) were produced by the new climate model INM-CM5 as an incremental upgrade of the INMCM4. We are mainly focusing on the question of how global mean surface temperature (GMST) changes are reproduced with the new forcing protocols proposed for CMIP6 and how these changes are connected with the reproduction of other features of the climate system mentioned above (i.e., $\mathrm{AMO}$ and $\mathrm{PDO}$ variability).

\section{Model and data}

The climate model INM-CM5 (Volodin et al., 2017a, b) was used in this study. In the atmosphere, it has a spatial resolution of $2 \times 1.5^{\circ}$ in longitude and latitude and 73 levels in the vertical, with the uppermost level at $0.2 \mathrm{hPa}$. In the oceanic block, the spatial resolution is $0.5 \times 0.25^{\circ}$ and 40 levels in the vertical. The model includes an interactive aerosol block (Volodin and Kostrykin, 2016), in which concentrations of 10 aerosols are calculated. In the numerical experiments discussed below only the first aerosol indirect effect (the influence of aerosol on cloud drop radius) is taken into consider- ation. A model description and analysis of simulations of the present day climate can be found in Volodin et al. (2017b).

Let us now discuss a climate change modeling experiment for years 1850-2014. Time series of $\mathrm{CO}_{2}, \mathrm{CH}_{4}, \mathrm{~N}_{2} \mathrm{O}, \mathrm{O}_{3}$, stratospheric volcanic sulfate aerosol concentration, total solar irradiance (TSI), and solar spectrum, as well as anthropogenic emissions of $\mathrm{SO}_{2}$, black carbon, and organic carbon were prescribed as recommended for the historical run of CMIP6 (Eyring et al., 2016). Seven model runs were started with different initial conditions obtained from a long preindustrial run, in which all external forcings were prescribed at the level of year 1850. The length of the preindustrial run was several hundred years, so the upper oceanic layer was adjusted to atmospheric model conditions, but it is not the case for the deep ocean. A small trend of model climate is visible because of deep ocean adjustment to upper oceanic and atmospheric conditions - a common situation for the simulation of historical climate with present day climate models. The obvious reason for multiple integrations is to separate the role of natural variability and external forcing in climate changes. When data from seven model runs are consistent with each other, then one can expect that the phenomenon of interest is a manifestation of (or response to) an external forcing. If there is a noticeable difference between different model runs, then a role of natural variability is crucial. To estimate the statistical significance of the near-surface temperature trend, a $t$ test at the $99 \%$ level was used. The variance of 5-year means was calculated from 1200 years of the preindustrial run.

Observational data of GMST for 1850-2014 used for verification of the model results were produced by HadCRUT4 (Morice et al., 2012). Monthly mean sea surface temperature (SST) data ERSSTv4 (Huang et al., 2015) are used for comparison of the AMO and PDO indices with that of the model. Data of Arctic sea ice extent for 1979-2014 derived from satellite observations are taken from Comiso and Nishio (2008). The stratospheric temperature trend and geographical distribution of near-surface air temperature trend for 19792014 are calculated from ERA-Interim reanalysis data (Dee et al., 2011).

\section{Results}

The most important measure of climate changes is the global mean surface temperature. Observed GMST demonstrates the well-known acceleration of warming in 1920-1940 and 1980-2000 and small warming or even small cooling in 1945-1970 and 2000-2014. The ensemble of CMIP5 models (Bindoff et al., 2013) shows less significant slowdown in warming in 2000-2014. In particular, the INMCM4 (Volodin et al., 2013) demonstrates gradual warming starting from 1920.

With the new CMIP6 protocols all seven INM-CM5 model runs demonstrate fast warming in 1980-2000 with a rate 


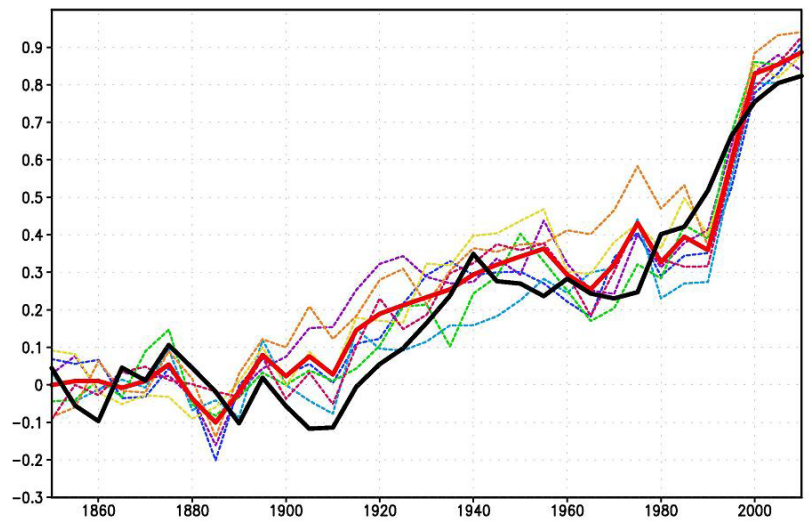

Figure 1. The 5-year mean GMST (K) anomaly with respect to 1850-1899 for HadCRUTv4 (thick solid black); model mean (thick solid red). Dashed thin lines represent data from individual model runs: 1 - purple, 2 - dark blue, 3 - blue, 4 - green, 5 - yellow, 6 - orange, 7 - magenta. In this and the next figures numbers on the time axis indicate the first year of the 5-year mean.

close to the observations and GMST stabilization in 20002014 and 1950-1970 (Fig. 1). The only significant difference in the new CMIP6 forcings at the beginning of the 21st century with respect to CMIP5 is the change in the TSI. Before year 2000 CMIP5 and CMIP6 TSI show almost identical behavior (CMIP5 solar forcing and other forcings are described in Taylor et al., 2012). In 2001-2008 the TSI recommended for CMIP6 is about $0.3 \mathrm{~W} \mathrm{~m}^{-2}$ lower than the one for CMIP5 (Fig. 2). For 2009-2014 the CMIP5 scenario suggested a repetition of the previous solar cycle that gives a value of the TSI almost $1 \mathrm{~W} \mathrm{~m}^{-2}$ above the one recommended for CMIP6. An additional model run with anthropogenic aerosol emissions fixed at the level of year 1850 shows a gradual GMST rise in 1950-1970 together with its stabilization in 2000-2014 (not shown). The latter fact supports the hypothesis that correct reproduction of GMST changes in 2000-2014 is due to the corrected CMIP6 treatment of the TSI. Another factor that stabilizes GMST in 2000-2014 in INM-CM5 is the heat flux to the ocean (Fig. 3) having values of $0.3-0.7 \mathrm{~W} \mathrm{~m}^{-2}$ (higher than in any period of the 20th century). The CMIP5 historical experiment with the INMCM4 shows a gradual increase in the ocean heat uptake during 1980-2005 rather than its abrupt jump in 1995-2005 seen in Fig. 3. Note that Yan et al. (2016) showed that according to the available observations, the slowdown in GMST increase in 1998-2013 can be explained by increased ocean heat uptake, which could be estimated as $0.7 \mathrm{~W} \mathrm{~m}^{-2}$ for $1993-2010$ according to Rhein et al. (2013).

The better representation of GMST stabilization in 19501970 (Fig. 1) in simulations with INM-CM5 with respect to the INMCM4 can be explained by the incorporation of a new aerosol block in the model that resulted in a more sophisticated treatment of anthropogenic and volcanic aerosol in-

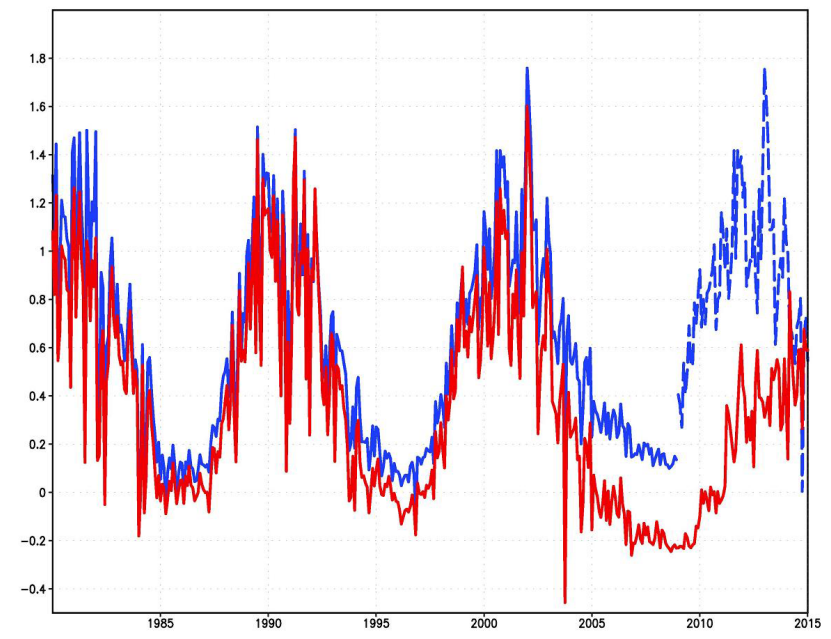

Figure 2. Monthly mean TSI anomaly $\left(\mathrm{W} \mathrm{m}^{-2}\right)$ with respect to 1882-1931 recommended for CMIP5 (blue; dashed line after year 2008 is the repetition of the data for 1998-2008) and for CMIP6 (red).

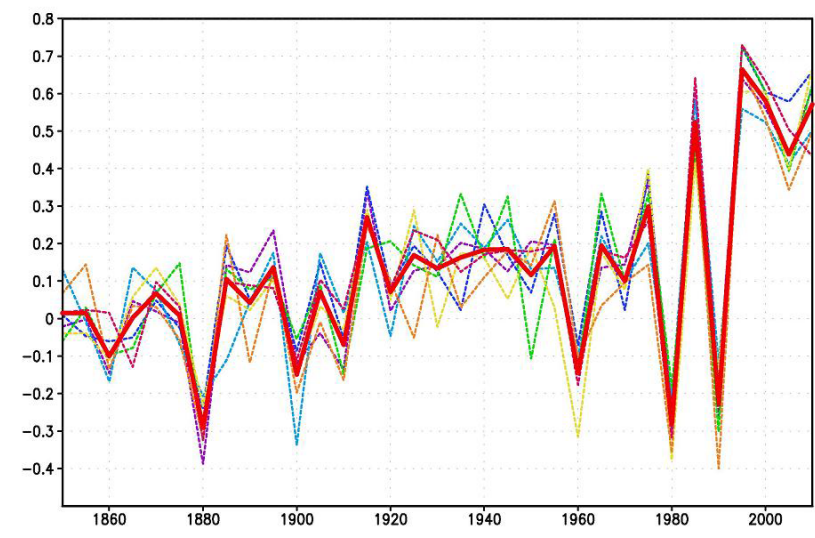

Figure 3. The 5-year mean surface heat flux; $\mathrm{W} \mathrm{m}^{-2}$ (positive downward). The thick solid red line represents model mean, and the dashed thin lines represent data from individual model runs: 1 purple, 2 - dark blue, 3 - blue, 4 - green, 5 - yellow, 6 - orange, 7 - magenta.

teraction with atmospheric radiation. Fast warming in 19201940 similar to observations can be seen in four model runs, while the other three runs show warming earlier or later. These results suggest that the observed acceleration of warming in 1920-1940 is probably due to a combination of external forcing and natural variability.

Keeping in mind the argument that the GMST slowdown in the beginning of the 21 st century could be due to the internal variability of the climate system, let us look at the behavior of the AMO and PDO climate indices. Here we calculated the AMO index in the usual way, as the SST anomaly in the Atlantic at latitudinal band $0-60^{\circ} \mathrm{N}$ minus the anomaly of the GMST. The model and observed 5-year mean AMO index 


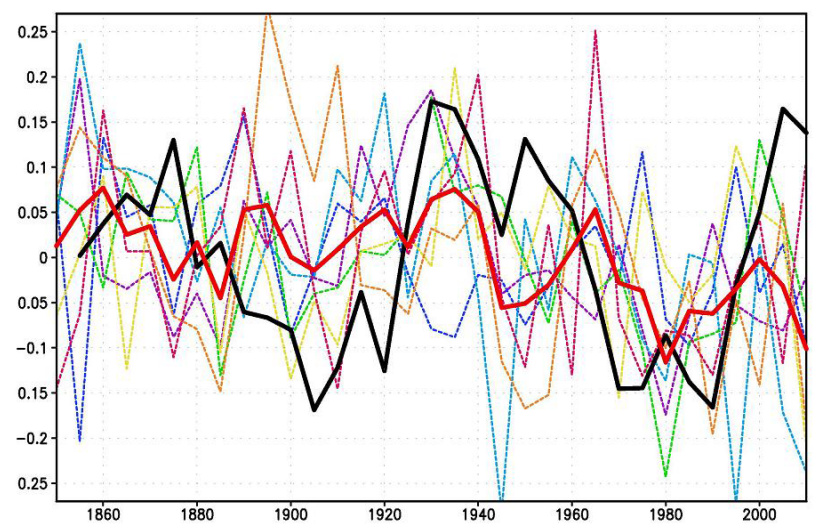

Figure 4. The 5-year mean AMO index (K) for ERSSTv4 data (thick solid black); model mean (thick solid red). Dashed thin lines represent data from individual model runs. Colors correspond to individual runs as in Fig. 1.

time series are presented in Fig. 4. The well-known oscillation with a period of 60-70 years can be clearly seen in the observations. Among the model runs, only one (dashed purple line) shows oscillation with a period of about 70 years, but without significant maximum near year 2000. In other model runs there is no distinct oscillation with a period of $60-70$ years but a period of $20-40$ years prevails. As a result none of the seven model trajectories reproduces the behavior of the observed AMO index after year 1950 (including its warm phase at the turn of the 20th and 21st centuries). One can conclude that anthropogenic forcing is unable to produce any significant impact on the AMO dynamics as its index averaged over seven realization stays around zero within one sigma interval (0.08). Consequently, the AMO dynamics are controlled by the internal variability of the climate system and cannot be predicted in historic experiments. On the other hand, the model can correctly predict GMST changes in 1980-2014 having the wrong phase of the AMO (blue, yellow, orange lines in Figs. 1 and 4).

More coherent behavior of model trajectories after year 1980 could be seen for the North Atlantic $\left(45-65^{\circ} \mathrm{N}\right)$ temperature (Fig. 5). Indeed, the temperature deviates from its 1850-1899 mean by 1.5 root mean square deviation in the early 2000s. The NA temperature index in the model shows notable oscillations with periods of about $30-40$ and 60 80 years (close to the 25 and 80 years for the observations), and three trajectories have correct strongly positive NA temperature anomalies in the 21 st century.

Another important climate feature that could be responsible for the changes in the GMST growth rate is the PDO measured by its index defined as the normalized projection of the SST anomaly on a specific pattern in the North Pacific at $20-60^{\circ} \mathrm{N}$. The 5-year average PDO index for observations and model data is presented in Fig. 6. For the observations, one can see maxima at years 1930-1940 and 1980-1995 and

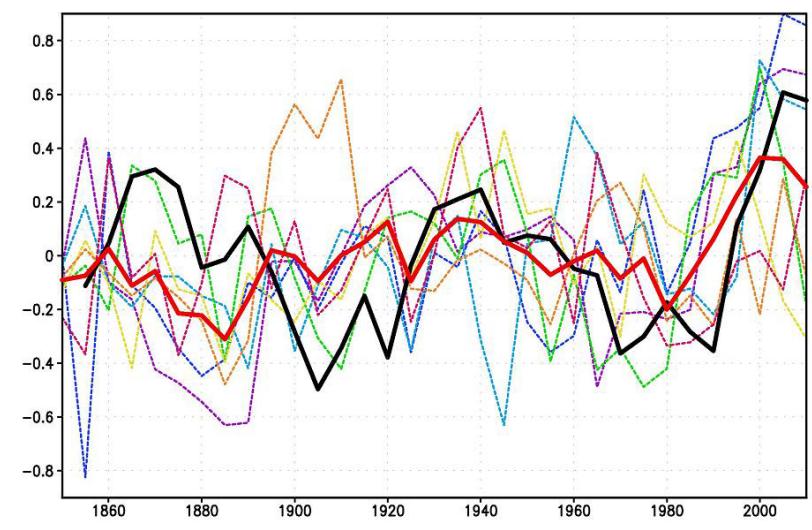

Figure 5. The 5-year mean SST anomaly (K) with respect to 1850 1899 in the North Atlantic $\left(45-65^{\circ} \mathrm{N}\right)$ for ERSSTv4 data (thick solid black); model mean (thick solid red). Dashed thin lines represent data from individual model runs. Colors correspond to individual runs as in Fig. 1.

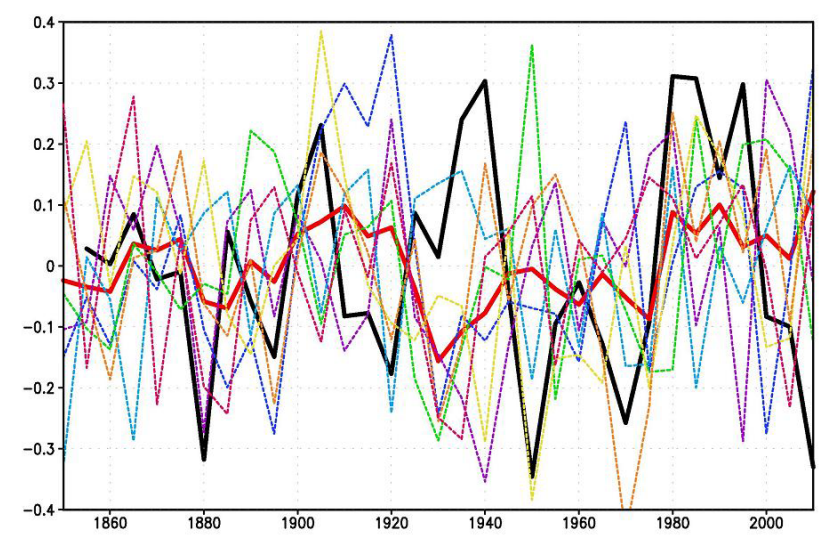

Figure 6. The 5-year mean PDO index (K) for ERSSTv4 data (thick solid black); model mean (thick solid red). Dashed thin lines represent data from individual model runs. Colors correspond to individual runs as in Fig. 1.

a prolonged minimum during 1950-1975. None of the model trajectories reflects observed time series of the PDO index for the same reasons discussed earlier in the paragraph devoted to the AMO. Again the model does not need correct PDO index dynamics to predict GMST behavior.

One of the most intriguing observed features of ongoing climate changes is the fast summer Arctic sea ice extent decrease in the beginning of the 21 st century. The ensemble of CMIP5 models underestimates the rate of decrease in Arctic summer ice area by a factor of 2. INMCM4 participated in CMIP5 and also significantly underestimates the extent of Arctic sea ice decrease (Volodin el al., 2013). In newly obtained INM-CM5 data (Fig. 7) we qualitatively see the same behavior of the Arctic sea ice as the average rate of sea ice loss is underestimated by a factor of 2 to 3 . However, in one model run (purple) the magnitude of decrease is similar to the 


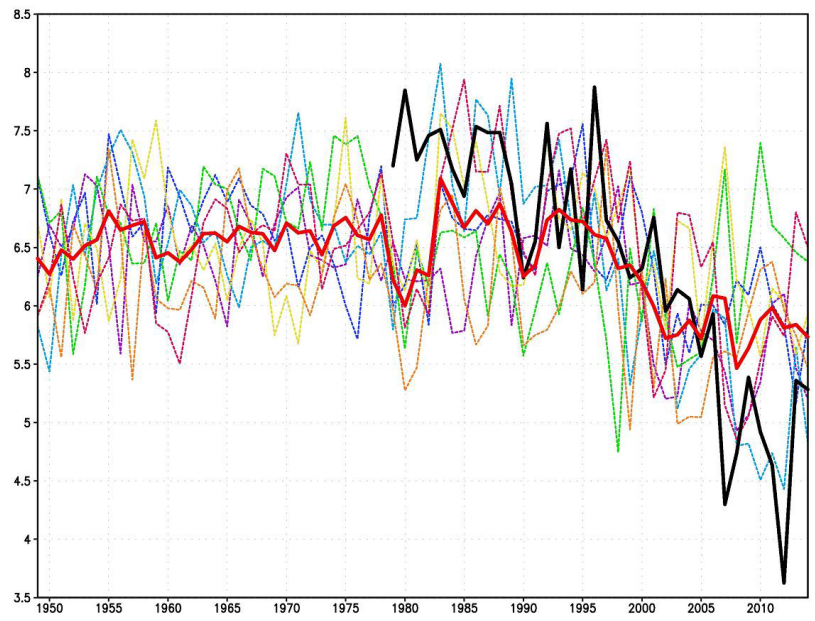

Figure 7. September Arctic sea ice extent $\left(10^{12} \mathrm{~m}^{2}\right)$ for observations (Comiso and Nishio, 2008) (thick solid black); model mean (thick solid red). Dashed thin lines represent data from individual model runs. Colors correspond to individual runs as in Fig. 1.

one in the observations (reduction from 7-7.5 million $\mathrm{km}^{2}$ in the 1980 s to $4-5.5$ million $\mathrm{km}^{2}$ in the $2000 \mathrm{~s}$ ). In other runs Arctic sea ice loss is underestimated by a factor of $1.5-3$, and in one run (green) one can even see some increase in Arctic sea ice area during the last decades. Our results suggest that the rapid decrease in Arctic sea ice extent near year 2000 was partially induced by external forcing; however, the role of internal variability can be very important (the range of the sea ice extent year-to-year variability could be estimated as 3.0 million $\mathrm{km}^{2}$ ).

The stratosphere is more sensitive to global changes than the troposphere. One can see in the observations stratospheric cooling by several degrees during the last decades. In the ERA-Interim reanalysis data (Fig. 8) the global and annual mean temperature at $5 \mathrm{hPa}$ in year 2014 is $3 \mathrm{~K}$ lower than in year 1979. All model runs show a gradual decrease in stratospheric temperature during all periods of the historical run from 1850 to 2014, but the rate of decrease in 1979-2014 is highest and equal to $2.5 \mathrm{~K}$, which is slightly below the absolute value observed. This strong decrease is consistent in all model runs and is likely produced by combined effects of $\mathrm{CO}_{2}$ increase and ozone decrease. Oscillations of global mean temperature at $5 \mathrm{hPa}$ with a period of $10-12$ years represent the prescribed solar cycle.

One of the characteristic features of climate changes in recent decades is a specific geographical pattern of surface temperature trends. Figure 9 shows the near-surface air temperature difference between 2000-2014 and 1985-1999 according to ERA-Interim reanalysis and model mean data. Statistical significance for model data was estimated using a $t$ test, and the $99 \%$ confidence level was used. Reanalysis data look noisier than model mean, but some observed features are reproduced well by the model ensemble. Maxi-

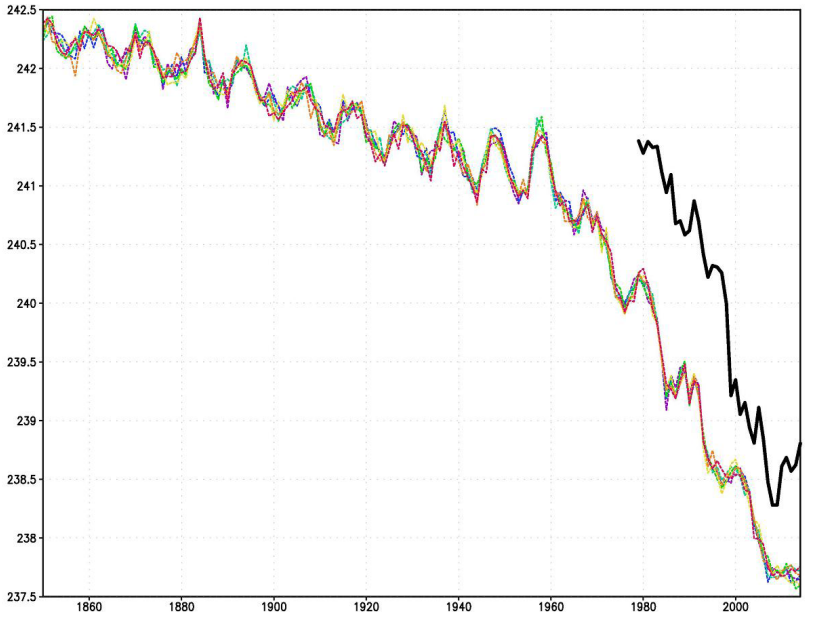

Figure 8. Annual mean global mean temperature $(\mathrm{K})$ at $5 \mathrm{hPa}$ for ERA-Interim data (black) and model data (dashed color lines).
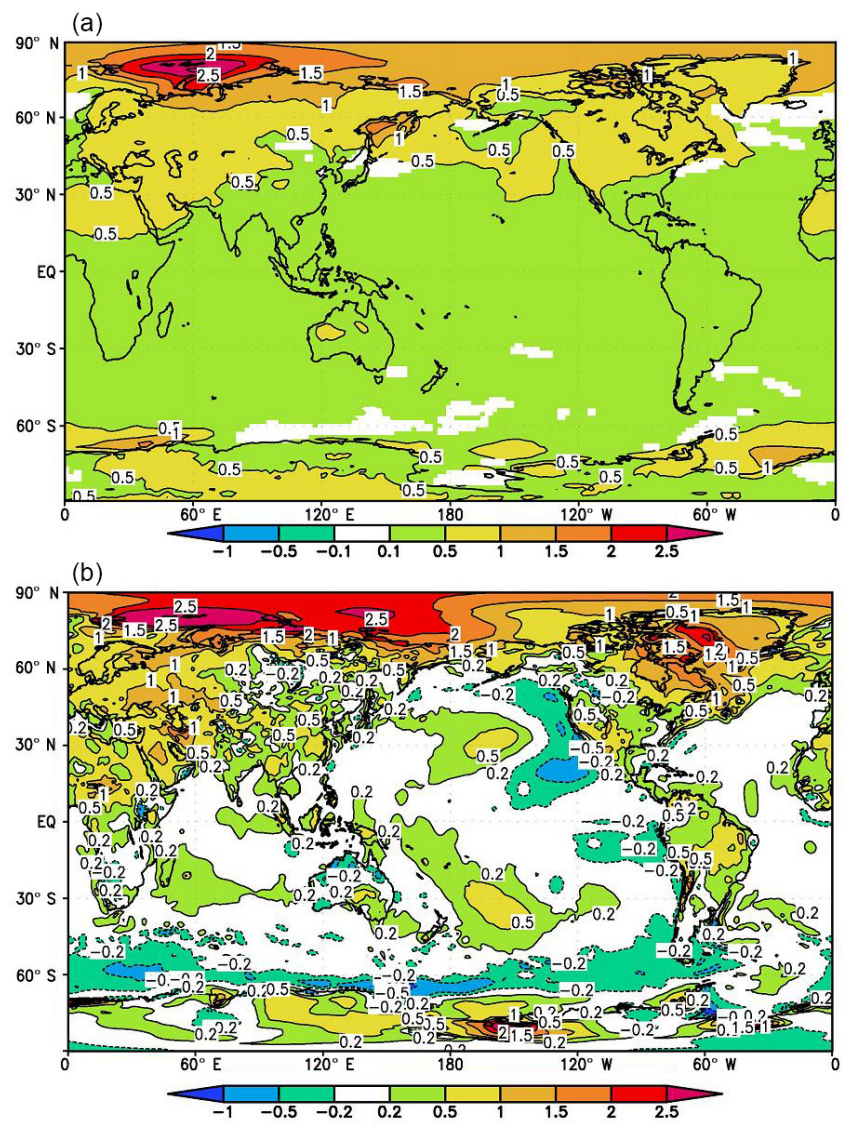

Figure 9. Annual mean near-surface air temperature (K) in 20002014 minus 1985-1999 for model mean data (a); shading represents the $99 \%$ level of significance; ERA-Interim data (b).

mum warming up to $2.5 \mathrm{~K}$ appears in the Arctic and in the Barents and Kara seas; warming over high and midlatitudes in Eurasia and North America is about $1 \mathrm{~K}$, with the low- 
est warming (or even cooling in reanalysis data) located in the Southern Ocean. Model warming is robust everywhere except some areas in the Southern Ocean, the zone of deep convection in the North Atlantic, and zones of Gulf Stream and Kuroshio separation from the shore, where natural variability is high. In the Pacific, the observed pattern connected with the PDO is not reproduced in model mean data or in any individual model run. Figure 10 represents the near-surface temperature model trend in two experiments (blue and green) with the maximum and minimum Arctic warming. In the second one (green) there is no Arctic warming at all and even some cooling, and warming over Eurasian and North American midlatitudes is also much smaller than in model average data. Otherwise, in the first case (blue) the Arctic warming in some areas is as large as $7 \mathrm{~K}$, and midlatitudinal warming over Eurasia and North America is higher than in model average data.

\section{Conclusions}

Seven historical runs for 1850-2014 with the climate model INM-CM5 were analyzed. It is shown that the magnitude of the GMST rise in model runs agrees with the estimate based on the observations. All model runs reproduce the stabilization of GMST in 1950-1970, fast warming in 1980-2000, and a second GMST stabilization in 2000-2014, suggesting that the major factor for predicting GMST evolution is the external forcing rather than system internal variability. $\mathrm{Nu}$ merical experiments with the previous model version (INMCM4) for CMIP5 showed unrealistic gradual warming in 1950-2014. The difference between the two model results could be explained by more accurate modeling of the stratospheric volcanic and tropospheric anthropogenic aerosol radiation effect (stabilization in 1950-1970) due to the new aerosol block in INM-CM5 and more accurate prescription of the TSI scenario (stabilization in 2000-2014) in the CMIP6 protocol. Four of seven INM-CM5 model runs simulate the acceleration of warming in 1920-1940 in a correct way; the other three produce it earlier or later than in reality. This indicates that for the warming during 1920-1940 the climate system natural variability plays a significant role.

No model trajectory reproduces the correct time behavior of the AMO and PDO indices. Taking into account our results on the GMST modeling one can conclude that anthropogenic forcing does not produce any significant impact on the dynamics of the AMO and PDO indices, at least for the INM-CM5 model. In turn, the correct prediction of the GMST changes in 1980-2014 and the increase in ocean heat uptake in 1995-2014 does not require correct phases of the AMO and PDO as all model runs have correct values of the GMST, while in at least three model experiments the phases of the AMO and PDO are opposite to the observed ones in that time. The variance explained by PDO and AMO is similar in the model and in the observations. The North Atlantic (a)

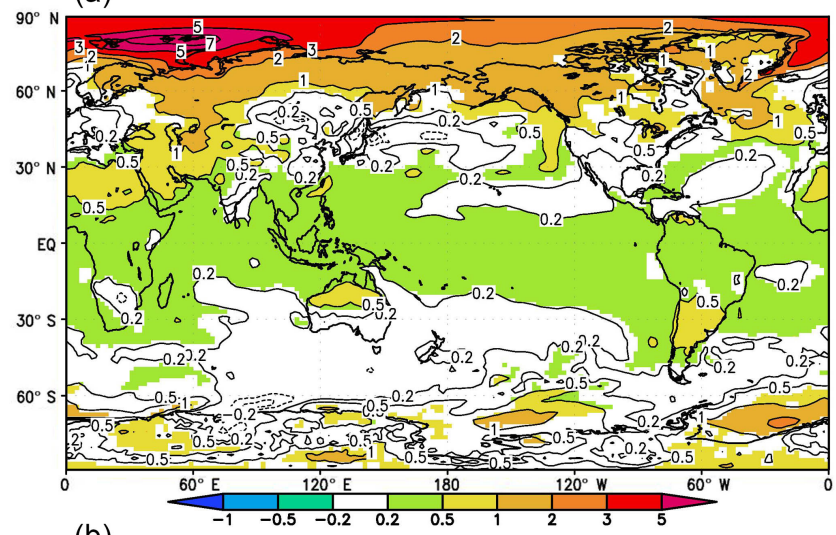

(b)

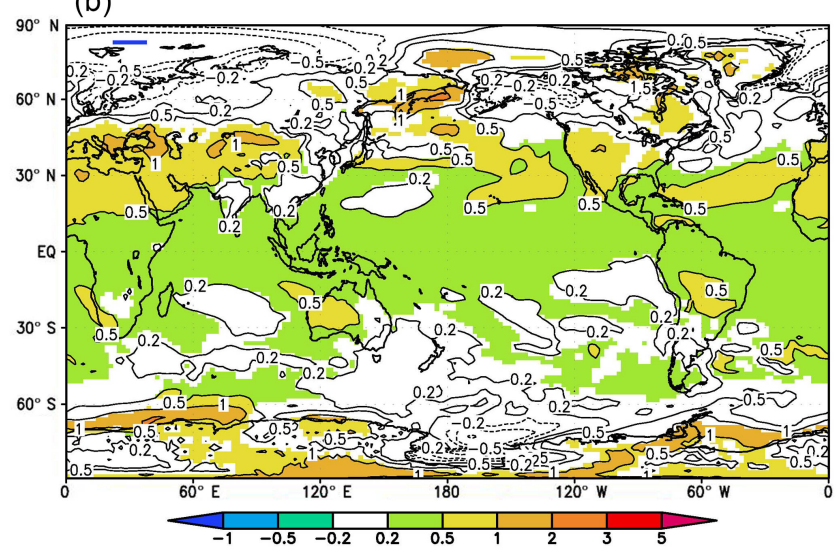

Figure 10. Annual mean near-surface air temperature $(K)$ in 2000 2014 minus 1985-1999 for the model run with highest (a) and lowest (b) warming in the Arctic. Shading represents the $99 \%$ level of significance.

SST time series produced by the model correlates better with the observations in 1980-2014. Three out of seven trajectories have a strongly positive North Atlantic SST anomaly as in the observations (in the other four cases we see near-tozero changes for this quantity).

The INM-CM5 has the same skill for prediction of the Arctic sea ice extent in 2000-2014 as CMIP5 models, including INMCM4. It underestimates the rate of sea ice loss by a factor between 2 and 3 . In one extreme case the magnitude of this decrease is as large as in the observations, while in the other the sea ice extent does not change compared to the preindustrial age. In part this could be explained by the strong internal variability of Arctic sea ice, but obviously the new version of INMCM and the new CMIP6 forcing protocol do not improve the prediction of the Arctic sea ice extent response to anthropogenic forcing.

The model reproduces several observed geographic features of the near-surface air temperature trend during the last decades, including Arctic amplification with a maximum over the Barents and Kara seas, warming of about $1 \mathrm{~K}$ over Eurasian and North American midlatitudes, and the weakest 
warming over the Southern Ocean. Case-to-case variability is very important here as well.

The decrease in stratospheric temperature at $5 \mathrm{hPa}$ during the period of 1979-2014 is successfully reproduced by the model in all experiments. The magnitude of the temperature drop is close to the one for ERA-Interim data (2.5 and $3 \mathrm{~K})$.

Data availability. Data on global mean near-surface temperature HadCRUT4 are available at https://crudata.uea.ac. uk/cru/data/temperature/ (last access: 30 March 2018). The oceanic temperature dataset ERSSTv4 can be downloaded at https://www1.ncdc.noaa.gov/pub/data/cmb/ersst/v4/netcdf/ (last access: 30 March 2018). The ERA-Interim reanalysis can be downloaded at https://www.ecmwf.int/en/forecasts/datasets/ archive-datasets/reanalysis-datasets/era-interim (last access: 30 March 2018). INM-CM5 model output is now available by request to the first author (volodinev@gmail.com), but will be added to the CMIP6 database.

Author contributions. AG produced model runs and collected model output; EV performed data processing.

Competing interests. The authors declare that they have no conflict of interest.

Acknowledgements. The study was performed at the Institute of Numerical Mathematics of the Russian Academy of Sciences and supported by the Russian Science Foundation, grant 14-27-00126. Climate model runs were produced with the supercomputer of the Joint Supercomputer Center of the Russian Academy of Sciences and supercomputer Lomonosov at Moscow State University.

Edited by: Christian Franzke

Reviewed by: two anonymous referees

\section{References}

Bindoff, N. L., Stott, P. A., AchutaRao, K. M., Allen, M. R., Gillett, N., Gutzler, D., Hansingo, K., Hegerl, G., Hu, Y., Jain, S., Mokhov, I. I., Overland J., Perlwitz, J., Sebbari, R., and Zhang, X.: Detection and Attribution of Climate Change: from Global to Regional, in: Climate Change 2013: The Physical Science Basis. Contribution of Working Group I to the Fifth Assessment Report of the Intergovernmental Panel on Climate Change, edited by: Stocker, T. F., Qin, D., Plattner, G.-K., Tignor, M., Allen, S. K., Boschung, J., Nauels, A., Xia, Y., Bex, V., and Midgley, P. M., Cambridge University Press, Cambridge, United Kingdom and New York, NY, USA, 2013.

Checa-Garcia, R., Shine, K. P., and Hegglin, M. I.: The contribution of greenhouse gases to the recent slowdown in globalmean temperature trends, Environ. Res. Lett., 11, 094018, https://doi.org/10.1088/1748-9326/11/9/094018, 2016.
Comiso, J. C. and Nishio, F.: Trends in the sea ice cover using enhanced and compatible AMSR-E, SSM/I, and SMMR data, J. Geophys. Res.-Oceans, 113, C02s07, https://doi.org/10.1029/2007JC004257, 2008.

Dee, D. P., Uppala, S. M., Simmons, A. J., Berrisford, P., Poli, P., Kobayashi, S., Andrae, U., Balmaseda, M. A., Balsamo, G., Bauer, P., Bechtold, P., Beljaars, A. C. M., van de Berg, L., Bidlot, J., Bormann, N., Delsol, C., Dragani, R., Fuentes, M., Geer, A. J., Haimberger, L., Healy, S. B., Hersbach, H., Holm, E. V., Isaksen, L., Kallberg, P., Kohler, M., Matricardi, M., McNally, A. P., Monge-Sanz, B. M., Morcrette, J. J., Park, B. K., Peubey, C., de Rosnay, P., Tavolato, C., Thepaut, J. N., and Vitart, F.: The ERA-Interim reanalysis: configuration and performance of the data assimilation system, Q. J. Roy. Meteorol. Soc., 137, 553597, https://doi.org/10.1002/qj.828, 2011.

Dong, L. and McPhaden, M. J.: The role of external forcing and internal variability in regulating global mean surface temperatures on decadal timescales, Environ. Res. Lett., 12, 034011, https://doi.org/10.1175/JCLI-D-17-0138.1, 2017.

Eyring, V., Bony, S., Meehl, G. A., Senior, C. A., Stevens, B., Stouffer, R. J., and Taylor, K. E.: Overview of the Coupled Model Intercomparison Project Phase 6 (CMIP6) experimental design and organization, Geosci. Model Dev., 9, 1937-1958, https://doi.org/10.5194/gmd-9-1937-2016, 2016.

Huang, B., Banzon, V. F., Freeman, E., Lawrimore, J., Liu, W., Peterson, T. C., Smith, T. M., Thorne, P. W., Woodruff, S. D., and Zhang, H. M.: Extended Reconstructed Sea Surface Temperature version 4 (ERSST.v4): Part I. Upgrades and intercomparisons, J. Climate, 28, 911-930, 2015.

McCusker, K. E., Fyfe, J. C., and Sigmond, M.: Twenty-five winters of unexpected Eurasian cooling unlikely due to Arctic sea-ice loss, Nat. Geosci., 9, 838-843, 2016.

Meehl, G. A., Arblaster, J. M., Fasullo, J. M., Hu, A., and Trenberth, K. E.: Model-based evidence of deep-ocean heat uptake during surface-temperature hiatus periods, Nature Clim. Change, 1, 360-364, 2011.

Morice, C. P., Kennedy, J. J., Rayner, N. A., and Jones, P. D.: Quantifying uncertainties in global and regional temperature changes using an ensemble of observational estimates: the HadCRUT4 dataset, J. Geophys. Res., 117, D08101, https://doi.org/10.1029/2011JD017187, 2011.

Overland, J. E., Wood, K. R., and Wang, M.: Warm Arctic-cold continents: climate impacts of the newly open Arctic Sea, Polar Res., 30, 15787, https://doi.org/10.3402/polar.v30i0.15787, 2011.

Rhein, M., Rintoul, S. R., Aoki, S., Campos, E., Chambers, D., Feely, R. A., Gulev, S., Johnson, G. C., Josey, S. A., Kostianoy, A., Mauritzen, C., Roemmich, D., Talley, L. D., and Wang, F.: Observations: Ocean, in: Climate Change 2013: The Physical Science Basis. Contribution of Working Group I to the Fifth Assessment Report of the Intergovernmental Panel on Climate Change, edited by: Stocker, T. F., Qin, D., Plattner, G.-K., Tignor, M., Allen, S. K., Boschung, J., Nauels, A., Xia, Y., Bex, V., and Midgley, P. M., Cambridge University Press, Cambridge, United Kingdom and New York, NY, USA, 2013.

Stroeve, J. C., Kattsov, V., Barrett, A., Serreze, M., Pavlova, T., Holland, M., and Meier, W. N.: Trends in Arctic sea ice extent from CMIP5, CMIP3 and observations, Geophys. Res. Lett., 39, L16502, https://doi.org/10.1029/2012GL052676, 2012. 
Taylor, K. E., Stouffer, R. J., and Meehl, G. A.: An overview of CMIP5 and the Experiment Design, B. Am. Meteorol. Soc., 93, 485-498, https://doi.org/10.1175/BAMS-D-11-00094.1, 2012.

Ting, M., Kushnir, Y., and Li, C.: North Atlantic Multidecadal SST Oscillation: External forcing versus internal variability, J. Marine Syst., 133, 27-38, 2014.

Volodin, E. M. and Kostrykin, S. V.: Aerosol block in climate model of INM RAS, Russ. Meteorol. Hydro., 8, 5-17, 2016.

Volodin, E. M., Diansky, N. A., and Gusev, A. V.: Simulation and Prediction of Climate Changes in the 19th to 21st Centuries with the Institute of Numerical Mathematics, Russian Academy of Sciences, Model of the Earth's Climate System. Izvestiya, Atmos. Ocean. Phys., 49, 347-366, 2013.

Volodin, E. M., Mortikov, E. V., Kostrykin, S. V., Galin, V. Y., Lykossov, V. N., Gritsun, A. S., Diansky, N. A., Gusev, A. V., and Iakovlev, N. G.: Simulation of modern climate with the new version of the INM RAS climate model, Izvestiya, Atmos. Ocean. Phys., 53, 142-155, 2017a.
Volodin, E. M., Mortikov, E. V., Kostrykin, S. V., Galin, V. Y., Lykossov, V. N., Gritsun, A. S., Diansky, N. A., Gusev, A. V., and Iakovlev, N. G.: Simulation of the present day climate with the climate model INMCM5, Clim. Dyn., V49, 3715, https://doi.org/10.1007/s00382-017-3539-7, $2017 \mathrm{~b}$.

Wilcox, L. J., Highwood, E. J., and Dunstone, N. J.: The influence of anthropogenic aerosol on multi-decadal variations of historical global climate, Environ. Res. Lett., 8, 024033, https://doi.org/10.1088/1748-9326/8/2/024033, 2013.

Yan, X. H., Boyer, T., Trenberth, K., Karl, T. R., Xie, S. P., Nieves, V., Tung, K. K., and Roemmich, D.: The global warming hiatus: Slowdown or redistribution?, Earth's Future, 4, 472-482, https://doi.org/10.1002/2016EF000417, 2016. 